systematisches Resümee gezogen wird. Der Rezensent hätte sehr gern mehr darüber erfahren, wie die Autorin, die die nordische Politik seit vier Jahrzehnten verfolgt, aus heutiger Perspektive die demokratischen Dynamiken einstuft und evaluiert. Warum erodieren im Norden die sozialdemokratischen Parteien? Weshalb verändern sich die Wettbewerbsdynamiken der nordischen Parteiensysteme? Und wieso ist gerade in den Kernlanden mächtiger Arbeiterbewegungen der moderne Rechtspopulismus so mächtig? Es hätte die Leser gewiss interessiert, welche theoretischen Schlussfolgerungen die Autorin aus ihren zahlreichen Studien und ihren langjährigen sozialwissenschaftlichen Analysen der nordischen Demokratien zieht. Ein systematisches Fazit anstelle eines kurzen Interviews hätte den sozialwissenschaftlichen Mehrwert der vorliegenden Studie beträchtlich gesteigert. Letztlich kann noch kritisiert werden, dass im ersten Kapitel (mit der Zählung „Null“) sowie im letzten Kapitel des Interviews viele redaktionelle Mängel und verwirrende Zeichensetzungen das Lesevergnügen mindern.

Insgesamt liegt hier ein beachtliches Dokument sozialwissenschaftlicher Forschungsbemühungen mit vielen interessanten Fakten vor. Zu bedauern ist jedoch die unzureichende theoretische Reflexion der Autorin über ihre ambitionierten Forschungsleistungen. Dieses Defizit macht den Band von Frauke Rubart auch nur bedingt anschlussfähig an Studien der politischen Mobilisierung in anderen Regionen Europas oder der Welt.

Sven Jochem

\title{
Entscheidungsfindung im Europäischen Parlament: aufschlussreiche Einblicke
}

Ringe, Nils: Who Decides, and How? Preferences, Uncertainty, and Policy Choice in the European Parliament, Oxford University Press, Oxford / New York 2010, 233 Seiten, € 55,-.

Als Legislative im Werden hat sich das Europäische Parlament (EP) und sein Innenleben in den vergangenen fünfzehn Jahren zu einem beliebten Testfeld für vielfältige methodische Zugänge, inhaltliche Schwerpunktsetzungen und forschungstheoretische Annahmen entwickelt. Die Monographie von Nils Ringe knüpft in ihrem Erkenntnisinteresse an das bisherige Referenzwerk „Democratic Politics in the European Parliament“von Simon Hix, Abdul G. Noury und Gérard Roland (2007) an. Sie will eine Erklärung für die Entscheidungsfähigkeit und die Ursachen empirisch beobachtbarer Abstimmungsgeschlossenheit der zum Teil heterogenen transnationalen Fraktionen liefern. In der bisherigen Literatur, die der Autor in Kapitel 1 konzise referiert, existieren dafür im Wesentlichen zwei Ansätze: Der erste erklärt Geschlossenheit bei Abstimmungen im Sinne von Kohäsion als normenund loyalitätsbasiertes Verhalten, das aus gemeinsamen Präferenzen und Wertorientierungen resultiert, der zweite unterstellt, dass Parteiführungen kraft ihrer Prärogative der Kandidatennominierung potenzielle Abweichler sanktionieren und disziplinieren können.

Ringe stellt beide Annahmen infrage. Seine Arbeit nimmt eine dezidiert mikrosoziologische Perspektive ein und hebt sich damit erfrischend von dem Beitrag von Hix u.a. ab. Im Zentrum stehen der individuelle Abgeordnete und die kausalen Mechanismen, die eine informierte Entscheidung auch ohne detaillierte inhaltliche Kenntnis des Abstimmungs- 
gegenstands gewährleisten. Ringe argumentiert plausibel, dass die auf Aggregatebene zu beobachtende Abstimmungsgeschlossenheit nichts über die dahinter stehenden Wirkungsmechanismen aussagt und im Grunde mit beiden Annahmen - Disziplin oder Kohäsion vereinbar ist. Sein Modell der „perceived preference coherence“ (PPC) ist erkennbar von der Principal-Agent-Theorie inspiriert. Es unterstellt eine Interdependenz von Präferenzen und Fraktionszugehörigkeit. Demnach gründet die Geschlossenheit der Fraktionen auf einer Delegationsbeziehung zwischen Experten und Nicht-Experten, die keiner Disziplinierung seitens einer Fraktionsführung bedarf: Experten in den ständigen Ausschüssen strukturieren die Meinungsbildung für ihre Fraktionen entscheidend vor, das heißt die Festlegung einer Fraktionslinie ist im Wesentlichen ein der Entscheidungsfindung im Ausschuss endogener Prozess. Nicht-Experten folgen den Empfehlungen ihrer sachkundigen Fraktionskollegen in der Regel in der Erwartung, dass diese am ehesten die eigenen Präferenzen teilen und in materielle Politik übersetzen können. Verstärkt wird dieser Effekt gleicher Fraktionszugehörigkeit noch, wenn es sich bei dem Experten um einen Abgeordneten gleicher nationaler Parteizugehörigkeit handelt, wie eine statistische Auswertung von 122 namentlichen Abstimmungen über legislative Entschließungen in der fünften Wahlperiode des EP (1999 bis 2004) belegt. Wenngleich dieser Befund an sich nicht neu ist, gebührt Ringe das Verdienst, die Erklärungen zu den Ursachen und den Wirkungsmechanismen hinter geschlossenem Abstimmungsverhalten in einem Modell kohärent zu systematisieren.

Den vielleicht wichtigsten Beitrag, den das Buch für die akademische Debatte liefert, bildet das Konzept der „focal points“. Es geht auf einen 2005 im American Journal of Political Science erschienenen Artikel des Autors zurück und bildet auch die Grundlage für die sechs Fallstudien, die im vorletzten Kapitel folgen und den kausalen Prozess hinter der Entscheidungsfindung mittels Interviewdaten plastisch machen. Focal points stellen sich als simplifizierte Darstellungen der Konsequenzen dar, die ein Gesetzgebungsvorschlag in der Praxis haben kann - in Bezug auf das Verhältnis zwischen EU und Nationalstaat, zwischen Markt und Staat oder in Bezug auf nationale wirtschaftliche Interessen. Das zentrale Argument lautet, dass insbesondere Abgeordnete, die über nur geringe Sachkenntnis verfügen, einem Gesetzentwurf aber grundsätzlich hohe Priorität beimessen, nicht völlig voraussetzungslos den Abstimmungsempfehlungen ihrer Ausschussexperten folgen, sondern eine Erläuterung verlangen, wie bestimmte Sachfragen mit den grundsätzlichen politischen Überzeugungen zusammenhängen.

Wie am Beispiel der Übernahmerichtlinie illustriert wird, kommt Berichterstattern eine Schlüsselposition bei der strategischen Anwendung solcher focal points zu: Durch geschickte Akzentuierung oder gar Überzeichnung bestimmter Aspekte und Zusammenhänge eines Legislativvorschlags lenken sie die Meinungsbildung nicht nur, sie können sogar Mehrheiten gegen ursprünglich mehrheitsfähige Positionen organisieren. Erhellend und modelltheoretisch stringent ist die Feststellung, dass externe Akteure wie Lobbygruppen oder nationale Regierungen im Ministerrat nur insoweit auf die parlamentsinterne Meinungsbildung einwirken, als Berichterstatter gleichsam als gate keeper Einwände und Kritik - in der Terminologie Ringes „competing focal points“ (S. 193) - aufgreifen und sie dies als Reaktion auf eine hinreichend breite „Alarmierung" von Nicht-Experten tun müssen. Ringe gelingt mit diesem theoretischen Konzept somit ein für die weitere empirische Forschung fruchtbarer Beitrag zur Erklärung, welche Rolle einzelne Schlüsselakteure in der Ausschusshierarchie für die prozessuale Steuerung eines Dossiers spielen.

Gleichwohl lässt das Buch einige Fragen unbeantwortet, die sich gerade angesichts jün- 
gerer Entwicklungen aufdrängen. So wäre es wünschenswert gewesen, die Bedeutung informeller Verfahren für die Gesetzgebung - gerade vor dem Hintergrund einer wachsenden Tendenz zu frühzeitigen Einigungen mit dem Rat - sowie die Konsequenzen für den Parteienwettbewerb näher zu beleuchten. Dies gilt umso mehr, als Ringe in seinem Literaturüberblick eine Reihe extrem interessanter Arbeiten ${ }^{1}$ zu diesem Thema aufführt und diesen Aspekt auch in einer der Fallstudien anreißt. Unberücksichtigt bleibt auch die zunehmende Praxis arbeitsteiliger Verfahren zwischen den Ausschüssen. Hierbei haben Berichterstatter des federführenden Ausschusses vor allem in der Frühphase des Gesetzgebungsprozesses nicht mehr so freie Hand in der Steuerung der parlamentarischen Beratung. Noch relevanter ist die Tatsache, dass Ausschüsse teilweise umgangen werden zugunsten informeller Foren, in denen vor allem Experten der beiden großen Fraktionen - Sozialdemokraten und Christdemokraten - eine maßgeblich Rolle für die Konsensbildung und Entscheidungsfindung spielen. ${ }^{2}$ Diese auch demokratietheoretisch wesentlichen Aspekte der Verfahrenssteuerung näher zu untersuchen muss folglich zukünftigen Arbeiten vorbehalten bleiben.

Nichtsdestoweniger ist Nils Ringe mit „Who Decides, and How?" eine theoretisch wie methodisch überzeugende und anspruchsvolle, zugleich anschauliche und gut lesbare Monographie gelungen, die dem Forschungsstand zum EP im Speziellen und zur Parlamentsforschung im Allgemeinen Substanzielles hinzuzufügen vermag, weil sie ihr zentrales Anliegen einlöst: eine Erklärung für die Ursachen hoher fraktioneller Geschlossenheit zu liefern, die am individuellen Abgeordneten und seiner Entscheidungsfähigkeit ansetzt.

Stefan Thierse

1 Vgl. Henry Farrell / Adrienne Héritier, Interorganizational Negotiation and Intraorganizational Power in Shared Decision Making: Early Agreements Under Codecision and Their Impact on the European Parliament and Council, in: Comparative Political Studies, 37. Jg. (2004), H. 10, S. $1184-1212$.

2 Vgl. Pierpaolo Settembri / Christine Neuhold, Achieving Consensus Through Committees: Does the European Parliament Manage?, in: Journal of Common Market Studies, 47. Jg. (2009), H. 1, S. $127-151$.

\section{Politikfelder der Europäischen Union: ideale Lektüre für Einsteiger}

Pollack, Johannes, Samuel Schubert und Peter Slominski: Die Energiepolitik der EU (Reihe Europa Kompakt, Band 5), Facultas, Wien 2010, 232 Seiten, € 19,50.

Puetter, Uwe: Die Wirtschafts- und Sozialpolitik der EU (Reihe Europa Kompakt, Band 3), Facultas, Wien 2010, 272 Seiten, € 19,50.

In den letzten Jahren wurden zahlreiche Bücher veröffentlicht, die sich mit der Europäischen Union (EU) befassen. Die meisten dieser Untersuchungen widmen sich der Frage, wie sich die institutionellen Strukturen auf Entscheidungssituationen auswirken beziehungsweise wie sich das europäische Mehrebenensystem auf die Politikgestaltung auswirkt. Vor diesem Hintergrund stellen die hier zu besprechenden Bücher aus der Reihe „Europa 